\title{
From Light to Heavy Nuclear Systems, Production and Decay of Fragments Studied with Powerful Arrays
}

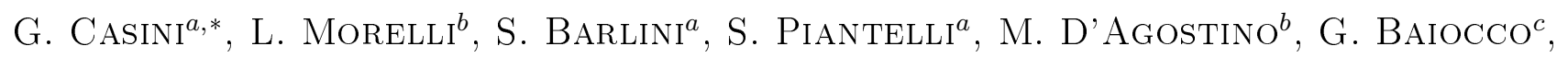

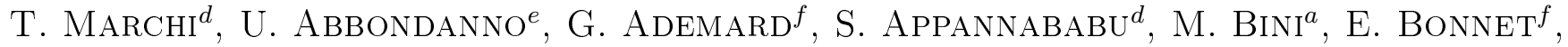
B. Borderie ${ }^{g}$, R. Bougault ${ }^{h}$, M. Bruno $^{b}$, A. Chbihi $^{f}$, M. Cinausero $^{d}$, M. Degerlier $^{i}$,

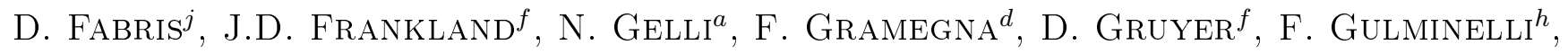

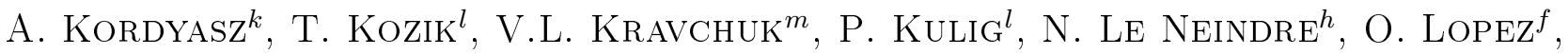

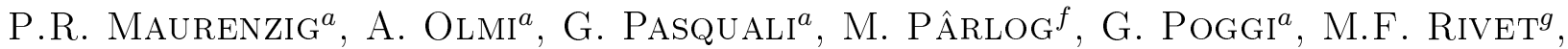

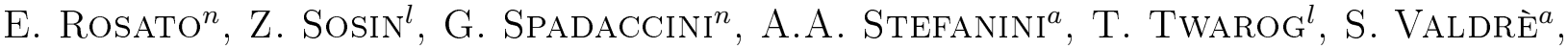
E. VIENT ${ }^{h}$ AND A.R. RADUTA ${ }^{o}$

${ }^{a}$ INFN Sezione di Firenze and Università di Firenze, Italy

${ }^{b}$ INFN Sezione di Bologna and Università di Bologna, Italy

${ }^{c}$ INFN Sezione di Pavia and Università di Pavia, Italy

${ }^{d}$ INFN-LNL Legnaro, Legnaro (Padova), Italy

${ }^{e}$ INFN, Sezione di Trieste, Trieste, Italy

${ }^{f}$ GANIL, CEA/DSM-CNRS/IN2P3, B.P. 5027, Caen, France

${ }^{g}$ Institut de Physique Nucléaire, CNRS/IN2P3, Université Paris-Sud 11, Orsay (Paris), France

${ }^{h}$ LPC, IN2P3-CNRS, ENSICAEN and Université de Caen, France

${ }^{i}$ Science and Art Faculty, Physics Department, H.B. Veli University, Nevsehir, Turkey

${ }^{j}$ INFN Sezione di Padova, Padova, Italy

${ }^{k}$ Heavy Ion Laboratory, University of Warsaw, Warsaw, Poland

${ }^{l}$ Institute of Physics, Jagiellonian University, Kraków, Poland

${ }^{m}$ National Research Center "Kurchatov Institute", Moscow, Russia

${ }^{n}$ INFN Sezione di Napoli and Università di Napoli, Italy

${ }^{\circ}$ NIPNE, Bucharest-Magurele, Romania

\begin{abstract}
Reactions between heavy-ions at various energy regimes produce many nuclear fragments which can be populated in highly excited states. The study of these fragments, detected at the end of their particle decay, is important to investigate nuclear forces and structure effects. In recent years there have been many efforts to extend these studies towards the drip-lines, i.e. to systems far from the $\beta$-stability valley, by using accelerated radioactive beams. The development of such infrastructures is accompanied by the development of more powerful detectors and associated electronics, capable to identify ions with very different sizes and kinetic energies. Here we give two examples which show how advanced arrays can contribute to the studies on nuclear phenomena. The examples come from the European FAZIA collaboration and from recent campaigns with the GARFIELD apparatus, the latter in operation at the INFN Legnaro Laboratory (Italy) where the SPES RIB facility is under construction.
\end{abstract}

DOI: 10.12693/APhysPolA.127.1548

PACS: 25.70.Gh, 25.70.Pq, 25.70.Mn, 29.40.-n

\section{Introduction}

Heavy-ion nuclear collisions, at different energy regimes, can produce a variety of fragments in a wide range of sizes and kinetic energies. The number and the type of these fragments depend also on the impact parameter. In general, with increasing bombarding energy and with decreasing impact parameter, we observe an

\footnotetext{
* corresponding author; e-mail: casini@fi.infn. it
}

increase of the product multiplicity and a reduction of their average sizes. For example, we mention the passage from the typical "U" shaped charge distribution of heavy fissioning nuclei at low energy to the powerlaw trend at Fermi energies, where many and relatively small fragments (intermediate mass fragments, IMF) are ejected [1]. Studying the features of these fragments is mandatory in order to understand the interaction mechanism and how the nuclear structure evolves when the systems are populated very far from the ground states. The investigation of the IMF's also impacts on other research fields. An example are the supernova explosive 
phases of core-collapse modeled using versions of the nuclear equation of state $E=E(\rho, \delta)$ where the isospin variable $\delta=\frac{(N-Z)}{N+Z}$ is tuned through our knowledge of the nuclei at extreme conditions. Another example are the light fragments produced beyond the Bragg peak in tissues when hadron beams of carbon or neon at 200$400 \mathrm{MeV} / \mathrm{u}$ are used in radiotherapy [2]: the knowledge of the differential cross-sections of various fragments in medical applications is mandatory to constrain treatment plan codes and to spare doses to healthy tissues.

The fragments produced in heavy-ion collisions are often excited and this excitation is relaxed through particle and gamma emission. Therefore, from an experimental side, the situation is even more complex and any attempt to access the primary nuclear distributions (those linked to the nuclear interaction processes) demands good detection techniques and refined analysis procedures.

In this contribution we deal with two subjects which are nowadays under investigation by several groups. The first one is the isospin $(N / Z)$ dynamics, in turn related to the symmetry energy term of the nuclear potential, whose behavior is not well constrained at high excitation and for systems with unusual neutron to proton $(N / Z)$ ratios. These studies can benefit from the use of radioactive beams, that allow to force the isospin degree of freedom, in particular favoring the population of n-rich nuclei. Correspondingly, experiments demand powerful detectors, capable of identifying the various reaction products in charge and mass.

A second interesting theme is the evidence of $\alpha$ structures in nuclei. It is well known that $\alpha$ particles represent a building block of many light nuclei. Many evidences exist of $\alpha$-states close to the ground-state of even self-conjugate nuclei; also, such structures, organized in different geometries, have been found to contribute to the properties of nuclei close to the separation energies or to the formation of molecular states in collisions between even $N=Z$ nuclei. However, the persistence of $\alpha$ cluster effects in strongly dissipative channels with formation of excited fused compounds has been only partially addressed at energies well above the particle separation.

Irrespective of the specific physics case, the development of efficient (with large acceptance) and powerful detectors is a common challenge and in this direction several activities are in progress worldwide. In the following we present some very recent results on fragment formation and decay, obtained with modern arrays on which an intense $R \& D$ or a proper upgrade have been carried out by our groups.

\section{Isospin dynamics explored with the FAZIA telescopes}

The European FAZIA collaboration [3] performed important developments on silicon-silicon-CsI(Tl) telescopes. This configuration is commonly used for heavyion studies. The focus has been put on the improvement of the ion identification capability of such devices, by acting both on detector construction and on electronics upgrading. In particular, the main idea was to improve the ion identification via pulse shape analysis (PSA) for fragments stopped in the first telescope layer, in order to lower the energy thresholds associated to the $\Delta E-E$ technique. The in beam tests on prototype telescopes indicated the relevant construction and mounting parameters which must be kept under control to reach sizeable improvements of the PSA. In general, the information on the particles impinging on the silicon diodes is associated with the time development of the charge (or current) signals. Therefore, any spurious effect which spoils the original signal shape must be reduced or avoided. Various effects and proposed solutions have been reported in many specific papers and are summarized in a recent review paper [4]. Here we remind that excellent results have been obtained with the test telescopes. Ions stopped in the first silicon layer were identified in charge via PSA up to $Z=54$, while the more critical mass separation was obtained up to the region of magnesium [5]. Also, the good quality of the entire devices and their electronics permitted isotopic separation of fast ions, punching through the first silicon, up to $Z=25[6,7]$. With such kind of telescopes, first experiments have been carried out at the Superconducting Cyclotron in LNS (Catania) aiming at accurate measurements of the IMF isotopic distributions. Beams of ${ }^{84} \mathrm{Kr}$ at $35 \mathrm{MeV} / \mathrm{u}$ impinged on ${ }^{112,124} \mathrm{Sn}$ thin targets. The quasi-projectile (QP) fragments were detected in small solid angles by our telescopes, mounted close to the grazing angle $\left(\approx 6^{\circ}\right)$ where most of the reaction cross-section is focused. The idea was to measure the isospin content of these QP fragments with varying the neutron richness of the target and in different phase space regions, from the QP one to the midvelocity zone. The results have been recently published [8] demonstrating the good performances obtained with our telescopes, which permitted to extend this kind of measurements (see for instance Ref. [9]) up to the "fission-like" fragments, in this case close to $Z=20$. Some of the results are summarized here.

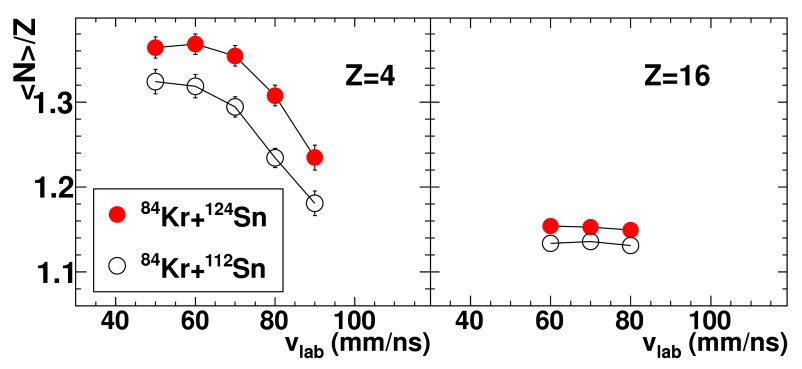

Fig. 1. Isospin ratios $\bar{N} / Z$ vs. the laboratory velocity for two fragments emitted at forward angles for the reactions ${ }^{84} \mathrm{Kr}+{ }^{112,124} \mathrm{Sn}$. Solid (open) symbols refer the n-rich (n-deficient) target isotope. Left part $Z=4$; right part $Z=16$.

Figure 1 reports, for two selected IMF species $Z=4$ and $Z=16$, the $\bar{N} / Z$ ratio versus the laboratory velocity. Only fragments which punch-through the first 
$300 \mu \mathrm{m}$ silicon layer of the telescope are retained, thus the $\Delta E-E$ technique is easily applied. Due to the high bombarding energy and the angular selection of QP ejectiles, this restriction does not affect the final results. In the figure we observe two aspects: (a) regardless of the velocity, the n-richness of IMF does depend on the target mass, pointing out that a certain isospin transport occurs in these collisions; (b) for both tin isotopes, fragments from the midvelocity region are more n-rich than fragments flying very closely to QP. There is an open debate on the origin of this second finding: the effect can be explained alternatively within statistical models including spin and Coulomb proximity effects [10] or by dynamical models where the dilute neck-region favors a neutron enrichment with respect to the surrounding QP and QT at normal density [11].

\section{3. $\alpha$-cluster effects in light compound nuclei at high excitation energies}

Several exclusive measurements have been performed by our INFN Nucl-ex collaboration to reveal signals of $\alpha$-clustering at high excitation energies in light nuclei. The idea is to detect deviations in the emitted particle properties, mainly $\alpha$ particles, with respect to the background of pure statistical emission from compound nuclei, CN. This approach has been already adopted; a recent application was reported [12] for the heavier ${ }^{48} \mathrm{Cr}$ compound nucleus at a lower excitation $(1.25 \mathrm{MeV} / \mathrm{u})$ than ours; moreover, the detecting system did not permit charge identification of the heavy fragments. The present study aims at a more complete detection in terms of acceptance and ion identification and focuses on lighter systems $(A=24)$. The GARFIELD [13] $\Delta E-E$ gasCsI(Tl) drift chambers, complemented with the forward Ring Counter telescopes recently upgraded [14], have been used to measure the fusion reactions ${ }^{12} \mathrm{C}+{ }^{12} \mathrm{C}$ and ${ }^{14} \mathrm{~N}+{ }^{10} \mathrm{~B}$, both leading to the $\alpha$-cluster ${ }^{24} \mathrm{Mg}$ nucleus, but in the former case both projectile and target are $\alpha$-cluster species. The beam-energy combinations were chosen in order to populate the compound system at the same excitation energy $(2.6 \mathrm{MeV} / \mathrm{u})$, well above the particle separation energies, where clustering features should fade out.

In the experiment, very exclusive events have been selected in which all charged particles (evaporation residues, ER, and light particles, LCP) have been detected and properly identified; this allows us for very stringent comparison between the two data sets. Each data set can be also compared with statistical model predictions, filtered through the geometry of the apparatus. In this way deviations from a pure statistical behavior can be evidenced and quantified.

Of course, a very fine tuning of the model parameters is needed in this approach and very constrained model predictions are crucial for signaling possible cluster effects. To this purpose, we decided to use for comparison not only statistical models available in the literature as the well-known GEMINI code [15] but also a purposely developed code. In the recent past our group implemented a version of the Hauser-Feshbach (H.F.) description of the evaporative decay, specifically tuned for light systems. Particular care has been taken to include the known energy levels (from available databases) of the various nuclides interested by the decay chains; moreover, a smooth transition has been inserted to link the level density parameter from the low energy where levels can be counted to the high region where only an average density can be assumed.

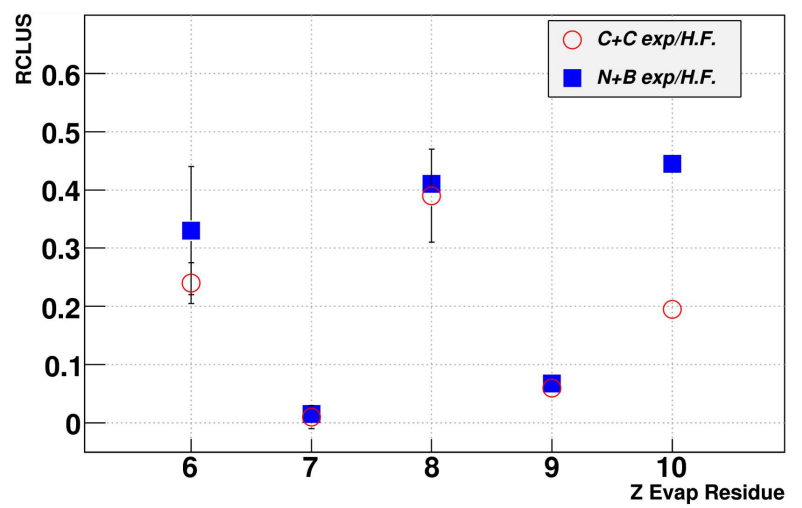

Fig. 2. $R_{\text {clus }}$ parameter as a function of the charge $Z$ of the ER for ${ }^{12} \mathrm{C}+{ }^{12} \mathrm{C}$ (open circles) and ${ }^{14} \mathrm{~N}+{ }^{10} \mathrm{~B}$ (solid squares). Predictions of the Hauser-Feshbach model have been used for both (systems see text).

Detailed results on the symmetric ${ }^{12} \mathrm{C}$ collision have been extensively published $[16,17]$ while the data analysis of the ${ }^{14} \mathrm{~N}+{ }^{10} \mathrm{~B}$ system is still in progress. Here we summarize one of the main obtained results in Fig. 2, where the parameter $R_{\text {clus }}$ is shown. It reads

$$
R_{\text {clus }}=\frac{\left|N^{\mathrm{e}}(Z)_{M}\right|}{N^{\mathrm{e}}(Z)}-\frac{\left|N^{\mathrm{c}}(Z)_{M}\right|}{N^{\mathrm{c}}(Z)},
$$

where $N^{\mathrm{e}, \mathrm{c}}(Z)_{M}$ is the number of events with an ER having charge $Z$ for which we measured the maximum allowable $\alpha$ multiplicity; $N^{\mathrm{e}, \mathrm{c}}(Z)$ is the total number of events with an ER having charge $Z$; the superscripts "e, c" label experimental and calculated quantities, respectively. $R_{\text {clus }}$ estimates the deviation of the $\alpha$ emission yield with respect to the statistical predictions as a function of the ER charge $Z$, for charge-complete events. Positive values of $R_{\text {clus }}$ indicate an excess of production, zero values means that data are explained by models. In Fig. 2 we use the Hauser-Feshbach code as the statistical decay reference. Clearly, for even- $Z$ channels, there is an effect which is not contained in the model. The effect is evident for the ${ }^{12} \mathrm{C}+{ }^{12} \mathrm{C}$ case, where error bars correspond to variations due to different cuts on the event $Q$-value (more or less damped events) [17]. Also in the case of ${ }^{14} \mathrm{~N}+{ }^{10} \mathrm{~B}$ we measure similar deviations with respect to the predicted branching ratios; the effect results even larger for the Ne channel for which the emission of the solely allowed $\alpha$ particle is definitely more favoured in the 
measurement. For this system the error bars correspond to different cuts on the maximum spin assumed for the compound nucleus and to slightly different selections on the experimental data set. The estimate of the deviations is under refinement, in particular for the ${ }^{14} \mathrm{~N}+{ }^{10} \mathrm{~B}$.

In summary, although the analysis is still in progress, we evidenced that $\alpha$-cluster effects persist at high excitation energies in $N=Z$ even compound nuclei; this effect appears to be associated not with the initial $\alpha$-structure of the reacting ions, but rather with that of the whole compound nucleus.

\section{References}

[1] N. Eren, N. Buyukcizmeci, R. Ogul, J. Phys. Conf. Series 436, 012063 (2013).

[2] E. Haettner, H. Iwase, D. Schardt, Radiat. Prot. Dosimetry 122, 485 (2006).

[3] FAZIA Collaboration web site, http://fazia2.in2p3.fr/spip.php?article2\&lang=en.

[4] R. Bougault, et al. (FAZIA Collaboration), Eur. Phys. J. A 50, 47 (2014).

[5] L. Bardelli, et al. (FAZIA Collaboration), Nucl. Instrum. Methods Phys. Res. A 654, 272 (2011).

[6] S. Carboni, et al. (FAZIA Collaboration), Nucl. Instrum. Methods Phys. Res. A 664, 251 (2012).

[7] N. Le Neindre, et al. (FAZIA Collaboration), Nucl. Instrum. Methods Phys. Res. A 701, 145 (2013).

[8] S. Barlini, et al. (FAZIA Collaboration), Phys. Rev. C 87, 054614 (2013).

[9] E. De Filippo, et al. (CHIMERA Collaboration), Phys. Rev. C 86, 014610 (2012).

[10] A. Ergun, H. Imal, N. Buyukcizmeci, R. Ogul, A.S. Botvina, arXiv:1408.2840, (2014).
[11] V. Baran, M. Colonna, V. Greco, M. Di Toro, Phys. Rep. 410, 335 (2005).

[12] E. Vardaci, A. Di Nitto, P. Nadtochy, A. Brondi, G. La Rana, R. Moro, A. Vanzanella, M. Cinausero, G. Prete, N. Gelli, J. Phys. Conf. Series 436, 012054 (2013).

[13] A. Moroni, M. Bruno, L. Bardelli, S. Barlini, S. Brambilla, G. Casini, R. Cavaletti, M. Chiari, A. Cortesi, M. D'Agostino, J. De Sanctis, E. Geraci, G. Giordano, A. Giussani, F. Gramegna, B. Guiot, V. Kravchuk, A. Lanchais, G.V. Margagliotti, A. Nannini, A. Ordine, S. Piantelli, G. Vannini, L. Vannucci, Nucl. Instrum. Methods Phys. Res. A 556, 516 (2006).

[14] M. Bruno, F. Gramegna, T. Marchi, L. Morelli, G. Pasquali, G. Casini, U. Abbondanno, G. Baiocco, L. Bardelli, S. Barlini, M. Bini, S. Carboni, M. Cinausero, M. D'Agostino, M. Degerlier, V.L. Kravchuk, E. Geraci, P.F. Mastinu, A. Ordine, S. Piantelli, G. Poggi, A. Moroni, Eur. Phys. J. A 49, 128 (2013).

[15] R.J. Charity, Phys. Rev. C 82, 014610 (2010).

[16] L. Morelli, G. Baiocco, M. D'Agostino, F. Gulminelli, M. Bruno, U. Abbondanno, S. Appannababu, S. Barlini, M. Bini, G. Casini, M. Cinausero, M. Degerlier, D. Fabris, N. Gelli, F. Gramegna, V.L. Kravchuk, T. Marchi, A. Olmi, G. Pasquali, S. Piantelli, S. Valdré, Ad R Raduta, J. Phys. G Nucl. Part. Phys. 41, 075107 (2014).

[17] L. Morelli, G. Baiocco, M. D'Agostino, F. Gulminelli, M. Bruno, U. Abbondanno, S. Appannababu, S. Barlini, M. Bini, G. Casini, M. Cinausero, M. Degerlier, D. Fabris, N. Gelli, F. Gramegna, V.L. Kravchuk, T. Marchi, A. Olmi, G. Pasquali, S. Piantelli, S. Valdré, Ad R Raduta, J. Phys. G Nucl. Part. Phys. 41, 075108 (2014) 